\title{
Nonlinear spectral singularities of a complex barrier potential and the lasing threshold condition
}

\author{
Ali Mostafazadeh* \\ Department of Mathematics, Koç University, Sarlyer 34450, Istanbul, Turkey
}

(Received 27 May 2013; published 24 June 2013)

\begin{abstract}
A spectral singularity is a mathematical notion with an intriguing physical realization in terms of certain zero-width resonances. In optics it manifests as lasing at the threshold gain. We explore the recently developed nonlinear generalization of spectral singularities for a complex barrier potential and study the associated resonance effect for an infinite planar slab of gain medium. In particular, for a Kerr nonlinearity, we show that the first-order perturbative equation that determines the nonlinear spectral singularities provides an explicit expression for the intensity of the emitted waves from the slab. This is a mathematical derivation of the known linear relationship between the output intensity of the slab and its gain coefficient. We also discuss the implications of our results for the time-reversed system which acts as a coherent perfect absorber.
\end{abstract}

DOI: $10.1103 /$ PhysRevA.87.063838

PACS number(s): 42.25.Bs, 03.65.Nk, 42.65.-k, 24.30.Gd

\section{INTRODUCTION}

One of the most interesting properties of complex scattering potentials [1] is that unlike their real analogs they can admit scattering states satisfying Siegert outgoing boundary conditions [2]. Because these states have a real and positive energy, they behave exactly like a zero-width resonance. This observation was originally made by the present author [3] in an attempt to understand the physical meaning of the mathematical notion of a spectral singularity [4]. The results obtained in this direction have motivated the study of the physical aspects and applications of spectral singularities, particularly in optics [3,5-15].

A remarkable outcome of this study is that for an optical system consisting of an infinite planar slab of homogeneous gain medium the well-known lasing threshold condition follows from the equation ensuring the generation of a spectral singularity [12]. Furthermore, if we consider the time-reversed setup, where the slab consists of a lossy medium with the loss coefficient having the same value as the gain coefficient required for a spectral singularity, it behaves as a coherent perfect absorber [11].

A more recent development is a nonlinear generalization of the notion of a spectral singularity [16]. A characteristic feature of nonlinear spectral singularities is that they are intensity dependent. This means that they correspond to the emission of waves with a particular wavelength-amplitude profile. In Ref. [16] we offer details of this phenomenon for a deltafunction potential with a complex coupling constant [17].

To improve our understanding of the properties and possible applications of nonlinear spectral singularities, in the present article, we study their behavior for a complex barrier potential that appears in modeling the interaction of electromagnetic waves with an infinite planar slab of gain material.

Consider a linearly polarized electromagnetic wave that is aligned along the $x$ axis in some Cartesian coordinate system and has an electric field of the form $\vec{E}=e^{-i \omega t} \mathscr{E}(z) \hat{e}_{x}$, where $\omega$ is the angular frequency of the wave, $\mathscr{E}$ is a complex-valued function, and $\hat{e}_{x}$ is the unit vector along the positive $x$ axis. Suppose that this wave interacts with an infinite planar slab of

*amostafazadeh@ku.edu.tr gain material of thickness $a$ that is aligned in the $x-y$ plane and located between the planes $z=0$ and $z=a$. Then it is well known that the Helmholtz equation for this system reduces to the Schrödinger equation

$$
-\mathscr{E}^{\prime \prime}(z)+V(z) \mathscr{E}(z)=k^{2} \mathscr{E}(z),
$$

for the energy-dependent complex barrier potential [12]

$$
V(z):=k^{2}\left(1-\mathfrak{n}^{2}\right) \chi_{a}(z),
$$

where $k:=\omega / c$ is the wave number, $\mathfrak{n}$ is the complex refractive index of the slab, and $\chi_{a}$ is the characteristic function of the interval $[0, a]$, i.e.,

$$
\chi_{a}(z):= \begin{cases}1 & \text { for } 0 \leqslant z \leqslant a, \\ 0 & \text { otherwise. }\end{cases}
$$

When a nonlinearity is present, $\mathfrak{n}^{2}$ is replaced by $\mathfrak{n}^{2}+$ $\sigma f(|\mathscr{E}(z)|)$ and (1) takes the form

$$
-\mathscr{E}^{\prime \prime}(z)+V(z) \mathscr{E}(z)-\sigma k^{2} \chi_{a}(z) f(|\mathscr{E}(z)|) \mathscr{E}(z)=k^{2} \mathscr{E}(z),
$$

where $\sigma$ is a real constant and $f$ is a real-valued function [18]. In terms of the scaled variables,

$$
\begin{aligned}
x & :=z / a, \quad \psi(x):=\mathscr{E}(a x), \quad \mathfrak{K}:=a k, \\
\gamma & :=-\mathfrak{K}^{2} \sigma, \quad \mathfrak{z}:=\mathfrak{K}^{2}\left(1-\mathfrak{n}^{2}\right), \quad v(x):=\mathfrak{z} \chi_{1}(x),
\end{aligned}
$$

we can write (4) as

$$
-\psi^{\prime \prime}(x)+v(x) \psi(x)+\gamma \chi_{1}(x) f(|\psi(x)|) \psi(x)=\mathfrak{K}^{2} \psi(x) .
$$

For a Kerr nonlinearity, where

$$
f(|\psi|)=|\psi|^{2},
$$

(6) is known as the Gross-Pitaevskii equation and has applications in the description of the Bose-Einstein condensates [19]. For real values of $\mathfrak{z}$ it admits exact analytic solutions in terms of the Jacobi elliptic functions [20].

\section{NONLINEAR SPECTRAL SINGULARITIES}

In Ref. [16] we introduced nonlinear spectral singularities associated with (6). In short, they correspond to the real and 
positive values of $\mathfrak{K}^{2}$ for which (6) admits a nonzero solution fulfilling the outgoing boundary conditions

$$
\psi^{\prime}(0)+i \mathfrak{K} \psi(0)=0, \quad \psi^{\prime}(1)-i \mathfrak{K} \psi_{k}(1)=0 .
$$

To see the physical implications of these conditions, we use the fact that for $x \notin[0,1]$ the general solution of (6) is a linear combination of the plane waves $e^{ \pm i \mathfrak{K} x}$. This allows us to construct scattering solutions of (6) corresponding to incident waves from the left $(x<0)$ and the right $(x>1)$. The former has the form [16]

$$
\psi^{l}(x)= \begin{cases}N_{-} e^{-i \mathfrak{K} x}+\tilde{N}_{-} e^{i \mathfrak{K} x} & \text { for } x<0, \\ \zeta(x) & \text { for } 0 \leqslant x \leqslant 1, \\ N_{+} e^{i \mathfrak{K} x} & \text { for } x>1,\end{cases}
$$

where $\zeta$ is a solution of (6) satisfying

$$
\zeta(1)=N_{+} e^{i \mathfrak{K}}, \quad \zeta^{\prime}(1)=i \mathfrak{K} N_{+} e^{i \mathfrak{K}},
$$

$N_{+}$is a complex constant determining the amplitude of the transmitted wave, and

$$
\begin{gathered}
N_{-}:=\frac{i G_{-}(\mathfrak{K})}{2 \mathfrak{K}}, \quad \tilde{N}_{-}:=-\frac{i G_{+}(\mathfrak{K})}{2 \mathfrak{K}}, \\
G_{ \pm}(\mathfrak{K}):=\zeta^{\prime}(0) \pm i \mathfrak{K} \zeta(0) .
\end{gathered}
$$

We can use (9) and (11) to derive the following expressions for the reflection and transmission coefficients from the left, respectively [16]:

$$
R^{l}=-\frac{G_{-}(\mathfrak{K})}{G_{+}(\mathfrak{K})}, \quad T^{l}=\frac{2 i k N_{+}}{G_{+}(\mathfrak{K})} .
$$

In view of (10) the solution (9) satisfies the second equation in (8). The first of these equations, which is equivalent to $G_{+}(\mathfrak{K})=0$, implies that both the reflection and transmission coefficients diverge. This is a characteristic property of a resonance state. Notice, however, that the energy $\mathfrak{K}^{2}$ does not have an imaginary part. Therefore (9) is a scattering solution of (6) which behaves like a resonance with a zero width [3].

Now suppose that we tune the parameters of the system so that it supports a spectral singularity with wave number $k$ and amplitude parameter $N_{+}$. Then any left-incident plane wave with wave number and amplitude, respectively, in a close vicinity of $k$ and $\tilde{N}_{-}$is amplified and emitted from both sides of the slab. In the absence of the nonlinearity, this phenomenon is amplitude independent and the system amplifies the background noise and serves as a laser that functions at the very threshold gain. This is because the mathematical expression describing the realization of a spectral singularity coincides with the laser threshold condition [12]. The presence of the nonlinearity modifies this expression by introducing in it a particular amplitude dependence. In the following we derive an explicit form of this expression and explain its physical meaning.

\section{LASING THRESHOLD CONDITION AND OUTPUT INTENSITY}

The determination of nonlinear spectral singularities requires the solution of the boundary-value problem defined by (6) and (8). This is equivalent to finding the function $\zeta$, which satisfies (6) and (10), and demanding that

$$
G_{+}(\mathfrak{K})=0 .
$$

For $x \in[0,1]$, we can express (6) in the form of the integral equation

$$
\psi(x)=\psi_{0}(x)+\gamma \int_{x_{0}}^{x} \mathscr{G}(x-y) f(|\psi(y)|) \psi(y) d y,
$$

where $\psi_{0}$ is the general solution of the linear equation

$$
\psi^{\prime \prime}+\mathfrak{n}^{2} \mathfrak{K}^{2} \psi=0,
$$

in $[0,1], x_{0} \in[0,1]$ is arbitrary, and $\mathscr{G}$ is the Green's function for this equation, i.e.,

$$
\mathscr{G}(u):=\frac{\sin (\mathfrak{n} \mathfrak{K} u)}{\mathfrak{n} \mathfrak{K}} .
$$

Repeated use of (15) in its right-hand side yields a perturbative series expansion for $\psi$ with $\gamma$ playing the role of the perturbation parameter. In particular, we find the following first-order perturbative expression for $\zeta$ :

$$
\zeta(x) \approx \zeta_{0}(x)+\gamma \zeta_{1}(x)
$$

where $\approx$ means that we neglect quadratic and higher-order terms in powers of $\gamma, \zeta_{0}$ is the solution of (16) satisfying (10), and

$$
\zeta_{1}(x):=\int_{1}^{x} \mathscr{G}(x-y) f\left(\left|\zeta_{0}(y)\right|\right) \zeta_{0}(y) d y .
$$

It is easy to show that

$\zeta_{0}(x)=\frac{N_{+} e^{i \mathfrak{K}}}{2 \mathfrak{n}}\left[(\mathfrak{n}+1) e^{i \mathfrak{n} \mathfrak{K}(x-1)}+(\mathfrak{n}-1) e^{-i \mathfrak{n} \mathfrak{K}(x-1)}\right]$.

Next, we insert (18) in (12) to obtain

$$
G_{ \pm}(\mathfrak{K}) \approx G_{ \pm}^{(0)}(\mathfrak{n}, \mathfrak{K})+\gamma G_{ \pm}^{(1)}(\mathfrak{n}, \mathfrak{K})
$$

where

$$
G_{ \pm}^{(j)}(\mathfrak{n}, \mathfrak{K}):=\zeta_{j}^{\prime}(0) \pm i \mathfrak{K} \zeta_{j}(0)
$$

for $j=0,1$. The computation of $G_{ \pm}^{(0)}(\mathfrak{K})$ is straightforward. It gives

where

$$
\begin{gathered}
G_{-}^{(0)}(\mathfrak{n}, \mathfrak{K})=\frac{N_{+} e^{i \mathfrak{K} \mathfrak{K}\left(\mathfrak{n}^{2}-1\right) \sin (\mathfrak{n} \mathfrak{K})}}{\mathfrak{n}}, \\
G_{+}^{(0)}(\mathfrak{n}, \mathfrak{K})=\left[\frac{i N_{+} e^{i(\mathfrak{n}+1) \mathfrak{K}}(\mathfrak{n}+1)^{2}}{2 \mathfrak{n}}\right] L(\mathfrak{n}, \mathfrak{K}),
\end{gathered}
$$

$$
L(\mathfrak{n}, \mathfrak{K}):=e^{-2 i \mathfrak{n} \mathfrak{K}}-\left(\frac{\mathfrak{n}-1}{\mathfrak{n}+1}\right)^{2} .
$$

According to (24) and (25), in the absence of nonlinearity, $\gamma=0$, and we find a spectral singularity provided that the right-hand side of (25) vanishes. This is in complete agreement with the results reported in Refs. [12-14].

Let $\mathfrak{n}_{0}$ and $\mathfrak{K}_{0}$ be such that $L\left(\mathfrak{n}_{0}, \mathfrak{K}_{0}\right)=0$, i.e., they correspond to a linear spectral singularity, and $\eta_{0}$ and $\kappa_{0}$ be, respectively, the real and imaginary parts of $\mathfrak{n}_{0}$, so that $\mathfrak{n}_{0}:=\eta_{0}+i \kappa_{0}$. Then in view of (25) and the fact that for typical gain media

$$
\eta_{0}-1 \gg-\kappa_{0}>0
$$


we have

$$
e^{-i \mathfrak{n}_{0} \mathfrak{K}_{0}}=\frac{\mathfrak{n}_{0}-1}{\mathfrak{n}_{0}+1} .
$$

Recalling that the gain coefficient $g_{0}$ necessary for the emergence of the spectral singularity is related to $\kappa_{0}$ according to $g_{0}=-2 \mathfrak{K}_{0} \kappa_{0} / a$, and taking the modulus square of both sides of (27), we obtain

$$
g_{0}=\frac{1}{2 a} \ln \frac{1}{|\mathcal{R}|^{2}},
$$

where $\mathcal{R}:=\left(\mathfrak{n}_{0}-1\right)^{2} /\left(\mathfrak{n}_{0}+1\right)^{2}$. In view of $(26), \mathcal{R} \approx\left(\eta_{0}-\right.$ $1)^{2} /\left(\eta_{0}+1\right)^{2}$. Therefore, it gives the reflexivity of the slab, and (28) coincides with the standard expression for the lasing threshold condition [12]

$$
g_{0} \approx \frac{2}{a} \ln \left(\frac{\eta_{0}+1}{\eta_{0}-1}\right)
$$

To obtain the nonlinear generalization of (29), we solve (14) perturbatively. Let $\mathfrak{n}_{1}$ and $\mathfrak{K}_{1}$ be such that, up to linear terms in $\gamma,(14)$ holds for

$$
\mathfrak{n}=\mathfrak{n}_{0}+\gamma \mathfrak{n}_{1}, \quad \mathfrak{K}=\mathfrak{K}_{0}+\gamma \mathfrak{K}_{1} .
$$

Inserting (30) in (21), expanding the resulting expression for $G_{+}$in powers of $\gamma$, and equating the coefficient of $\gamma$ to zero give

$$
\partial_{\mathfrak{n}_{0}} G_{+}^{(0)}\left(\mathfrak{n}_{0}, \mathfrak{K}_{0}\right) \mathfrak{n}_{1}+\partial_{\mathfrak{K}_{0}} G_{+}^{(0)}\left(\mathfrak{n}_{0}, \mathfrak{K}_{0}\right) \mathfrak{K}_{1}+G_{+}^{(1)}\left(\mathfrak{n}_{0}, \mathfrak{K}_{0}\right)=0 .
$$

Equation (27) simplifies the calculation of $\partial_{\mathfrak{n}_{0}} G_{+}^{(0)}\left(\mathfrak{n}_{0}, \mathfrak{K}_{0}\right)$ and $\partial_{\mathfrak{K}_{0}} G_{+}^{(0)}\left(\mathfrak{n}_{0}, \mathfrak{K}_{0}\right)$ enormously. The result is

$$
\begin{gathered}
\partial_{\mathfrak{n}_{0}} G_{+}^{(0)}\left(\mathfrak{n}_{0}, \mathfrak{K}_{0}\right)=\frac{N_{+} e^{i \mathfrak{K}_{0}} \mathfrak{K}_{0}\left[\left(\mathfrak{n}_{0}^{2}-1\right) \mathfrak{K}_{0}-2 i\right]}{\mathfrak{n}_{0}}, \\
\partial_{\mathfrak{K}_{0}} G_{+}^{(0)}\left(\mathfrak{n}_{0}, \mathfrak{K}_{0}\right)=N_{+} e^{i \mathfrak{K}_{0}} \mathfrak{K}_{0}\left(\mathfrak{n}_{0}^{2}-1\right) .
\end{gathered}
$$

The calculation of $G_{+}^{(1)}\left(\mathfrak{n}_{0}, \mathfrak{K}_{0}\right)$ is more involved. However, it turns out that we can use (19), (22), and (27) to write it in the form

$$
G_{+}^{(1)}\left(\mathfrak{n}_{0}, \mathfrak{K}_{0}\right)=-N_{+} e^{i \mathfrak{K}_{0}} c_{\mathfrak{n}_{0}}^{2} \int_{0}^{1} f\left(\left|N_{+} c_{\mathfrak{n}_{0}} h(x)\right|\right) h(x)^{2} d x,
$$

where

$$
c_{\mathfrak{n}_{0}}:=\frac{\mathfrak{n}_{0}+1}{2 \mathfrak{n}_{0}}, \quad h(x):=e^{i \mathfrak{n}_{0} \mathfrak{K}_{0}(x-1)}+e^{-i \mathfrak{n}_{0} \mathfrak{K}_{0} x} .
$$

For a Kerr nonlinearity (7), we can easily perform the integral in (34) and use (27) to express $G_{+}^{(1)}\left(\mathfrak{n}_{0}, \mathfrak{K}_{0}\right)$ as

$$
G_{+}^{(1)}\left(\mathfrak{n}_{0}, \mathfrak{K}_{0}\right)=\frac{8 i\left|N_{+}\right|^{2} N_{+} e^{i \mathfrak{K}_{0}}\left(4 \mathfrak{n}_{0}^{2}-\mathfrak{n}_{0}^{* 2}-3\right)}{\mathfrak{K}_{0}\left(9 \mathfrak{n}_{0}^{4}+\mathfrak{n}_{0}^{* 4}-10\left|\mathfrak{n}_{0}\right|^{4}\right)} .
$$

We can determine the nonlinear corrections to the location of the spectral singularities by substituting (32), (33), and (35) in (31) and solving the resulting equation for any two of $\operatorname{Re}\left(\mathfrak{n}_{1}\right), \operatorname{Im}\left(\mathfrak{n}_{1}\right)$, and $\mathfrak{K}_{1}$ in terms of the third.

To gain a better understanding of the consequences of (31), we expand the right-hand sides of (32), (33), and (35) in powers of $\kappa_{0}$ and keep the leading-order term. This gives

$$
\begin{gathered}
\partial_{\mathfrak{n}_{0}} G_{+}^{(0)}\left(\mathfrak{n}_{0}, \mathfrak{K}_{0}\right) \approx \frac{N_{+} e^{i \mathfrak{K}_{0}} \mathfrak{K}_{0}\left[\left(\eta_{0}^{2}-1\right) \mathfrak{K}_{0}-2 i\right]}{\eta_{0}}, \\
\partial_{\mathfrak{K}_{0}} G_{+}^{(0)}\left(\mathfrak{n}_{0}, \mathfrak{K}_{0}\right) \approx N_{+} e^{i \mathfrak{K}_{0}} \mathfrak{K}_{0}\left(\eta_{0}^{2}-1\right), \\
G_{+}^{(1)}\left(\mathfrak{n}_{0}, \mathfrak{K}_{0}\right) \approx \frac{3\left|N_{+}\right|^{2} N_{+} e^{i \mathfrak{K}_{0}}\left(\eta_{0}^{2}-1\right)}{4 \eta_{0}^{3} \mathfrak{K}_{0} \kappa_{0}} .
\end{gathered}
$$

We also note that the gain coefficient $g$ required to support a nonlinear spectral singularity is given by

$$
g=-\frac{2 \mathfrak{K} \kappa}{a} \approx g_{0}\left[1+\gamma\left(\frac{\kappa_{1}}{\kappa_{0}}+\frac{\mathfrak{K}_{1}}{\mathfrak{K}_{0}}\right)\right],
$$

where $\kappa:=\operatorname{Im}(\mathfrak{n})$ and $\kappa_{1}:=\operatorname{Im}\left(\mathfrak{n}_{1}\right)$.

Now suppose that we are interested in a nonlinear spectral singularity corresponding to a Kerr nonlinearity that has the same wavelength as its linear analog. Then $\mathfrak{K}_{1}=0$ and (31) together with (36) to (39) give

$$
\begin{aligned}
g & \approx g_{0}\left[1-\frac{6\left(\eta_{0}^{2}-1\right) \gamma\left|N_{+}\right|^{2}}{\eta_{0}^{2}\left[\left(\eta_{0}^{2}-1\right)^{2} \mathfrak{K}_{0}^{2}+4\right] \ln ^{2}\left(\frac{\eta_{0}+1}{\eta_{0}-1}\right)}\right] \\
& \approx g_{0}\left[1+\frac{6 \sigma\left|N_{+}\right|^{2}}{\eta_{0}^{2}\left(\eta_{0}^{2}-1\right) \ln ^{2}\left(\frac{\eta_{0}+1}{\eta_{0}-1}\right)}\right] .
\end{aligned}
$$

Here in the last relation we have switched to the unscaled nonlinearity parameter $\sigma$ and used the fact that for typical optical setups $\left(\eta_{0}^{2}-1\right)^{2} / \mathfrak{K}_{0}^{2} \approx 0$.

Equation (40) implies that for a positive Kerr nonlinearity parameter $\sigma$, which is usually the case [18], the presence of the nonlinearity increases the necessary gain for producing a spectral singularity. More importantly, it shows that the slab acts as an amplifier for left-incident plane waves only if $g$ exceeds $g_{0}$. In this case it emits an amplified transmitted plane wave whose intensity is given by

$$
\frac{1}{2}\left|N_{+}\right|^{2} \approx\left(\frac{g-g_{0}}{12 \sigma g_{0}}\right)\left[\eta_{0}^{2}\left(\eta_{0}^{2}-1\right) \ln ^{2}\left(\frac{\eta_{0}+1}{\eta_{0}-1}\right)\right] .
$$

Because this relation follows from our first-order perturbative analysis, it applies for situations where $\sigma\left|N_{+}\right|^{2} \ll 1$. Typically, $\sigma<10^{-13} \mathrm{~cm}^{2} / \mathrm{W}$ which for a value of $\left|N_{+}\right|^{2}$ as large as $1 \mathrm{GW} / \mathrm{cm}^{2}$ gives $\sigma\left|N_{+}\right|^{2}<10^{-4}$. This shows that (41) is quite reliable.

We can view (41) as a mathematical justification for the well-known fact that lasers operate when the gain coefficient is larger than $g_{0}$. It is important to notice that in the linear case the spectral singularity disappears as the value of the gain coefficient exceeds $g_{0}$ and the slab stops functioning as a laser. This is not the case when we take into account the effect of a Kerr nonlinearity with $\sigma>0$.

We have performed a first-order perturbative calculation of $G_{-}(\mathfrak{K})$ and used the result together with (11) to compute the amplitude parameter $N_{-}$for the emitted wave from the left-hand side of the slab. This calculation shows that whenever we arrange the parameters of the system to generate a nonlinear spectral singularity, the contribution of the terms of the order $\gamma$ to $N_{-}$cancel one another and we find $N_{-} \approx N_{+} e^{i \mathfrak{K}}$. Therefore similarly to the linear case, the wave emitted from the left-hand side of the slab has the same amplitude and phase as the one emitted from its right-hand side. This is a manifestation of the 
$\mathcal{P}$ symmetry of nonlinear spectral singularities established in Ref. [16]. For the time-reversed system, it implies that a lossy slab serves as a coherent perfect absorber provided that the loss factor $\alpha$ has a numerical value that is larger than $g_{0}$ and the intensity $\left|N_{+}\right|^{2} / 2$ of the incident coherent waves is given by (41) with $g$ replaced with $\alpha$.

\section{CONCLUDING REMARKS}

An interesting outcome of the search for the physical implications of spectral singularities is a mathematical derivation of the lasing threshold condition for an infinite planar slab of gain material [12]. This is quite different from the standard derivation of this condition that is essentially based on the physical principle of conservation of energy [21]. It involves determining the linear spectral singularities of a complex barrier potential. In the present article we have addressed the problem of locating nonlinear spectral singularities of this potential and explored the physical consequences of their emergence.

Our method is quite general and can be applied to almost all nonlinearities of physical interest. For a Kerr nonlinearity it provides a simple mathematical verification of the well-known fact that the slab model we consider does not function as a laser at the threshold gain $g_{0}$. It starts emitting radiation only when the gain coefficient exceeds $g_{0}$. More specifically, the first-order perturbative treatment of the nonlinear spectral singularities of this model gives rise to a purely mathematical derivation of an expression for the intensity of the emitted radiation. This expression agrees with the well-known results obtained using physical considerations, thus confirming the remarkable relevance of the mathematics of spectral singularities and their nonlinear generalization to the physics of lasers. This is another striking example of what Wigner once called "the unreasonable effectiveness of mathematics in natural sciences."

\section{ACKNOWLEDGMENTS}

We wish to thank Aref Mostafazadeh, Alphan Sennaroğlu, and Ali Serpengüzel for useful discussions. This work has been supported by the Scientific and Technological Research Council of Turkey (TÜBITAK) in the framework of the Project No. 110T611, and by the Turkish Academy of Sciences (TÜBA).
[1] J. G. Muga, J. P. Palao, B. Navarro, and I. L. Egusquiza, Phys. Rep. 395, 357 (2004).

[2] A. J. F. Siegert, Phys. Rev. 56, 750 (1939).

[3] A. Mostafazadeh, Phys. Rev. Lett. 102, 220402 (2009).

[4] M. A. Naimark, Trudy Moskov. Mat. Obsc. 3, 181 (1954); [Amer. Math. Soc. Transl. (2), 16, 103 (1960)]; G. Sh. Guseinov, Pramana. J. Phys. 73, 587 (2009), and references therein.

[5] A. Mostafazadeh, Phys. Rev. A 80, 032711 (2009).

[6] Z. Ahmed, J. Phys. A 42, 472005 (2009).

[7] S. Longhi, Phys. Rev. B 80, 165125 (2009); Phys. Rev. A 81, 022102 (2010).

[8] B. F. Samsonov, J. Phys. A 44, 392001 (2011); Phil. Trans. R. Soc. A 371, 20120044 (2013).

[9] A. Mostafazadeh, J. Phys. A 44, 375302 (2011); 45, 444024 (2012); A. Mostafazadeh and M. Sarisaman, Phys. Lett. A 375, 3387 (2011); Proc. R. Soc. A 468, 3224 (2012).

[10] F. Correa and M. S. Plyushchay, Phys. Rev. D 86, 085028 (2012).

[11] S. Longhi, Physics 3, 61 (2010); Phys. Rev. A 82, 031801 (2010); 83, 055804 (2011).
[12] A. Mostafazadeh, Phys. Rev. A 83, 045801 (2011).

[13] A. Mostafazadeh, Phys. Rev. A 84, 023809 (2011).

[14] A. Mostafazadeh and S. Rostamzadeh, Phys. Rev. A 86, 022103 (2012).

[15] L. Chaos-Cador and G. García-Calderón, Phys. Rev. A 87, 042114 (2013).

[16] A. Mostafazadeh, arXiv:1303.2501 [Phys. Rev. Lett. (to be published)].

[17] A. Mostafazadeh, J. Phys. A: Math. Gen. 39, 13495 (2006); H. F. Jones, Phys. Rev. D 76, 125003 (2007); A. Mostafazadeh and H. Mehri-Dehnavi, J. Phys. A 42, 125303 (2009).

[18] P. W. Milonni and J. H. Eberly, Laser Physics (Wiley, Hoboken, NJ, 2010).

[19] K. Rapedius, D. Witthaut, and H. J. Korsch, Phys. Rev. A 73, 033608 (2006).

[20] L. D. Carr, C. W. Clark, and W. P. Reinhardt, Phys. Rev. A 62, 063610 (2000)

[21] W. T. Silfvast, Laser Fundamentals (Cambridge University Press, Cambridge, England, 1996). 